\title{
Spectroscopy and imaging of newly discovered planetary nebulae
}

\author{
F. Kerber, G. Lercher and R. Weinberger \\ Institut für Astronomie der Leopold-Franzens-Universität Innsbruck, Technikerstraße 25, A-6020 Innsbruck, Austria \\ http://ast7.uibk.ac.at
}

Received January 3; accepted March 22, 1996

\begin{abstract}
The major purpose of this paper was to prove or disprove the nature of planetary nebula (PN) candidates in the northern hemisphere, taken from an internal list. We present spectroscopic observations and imaging of fifteen PNe, twelve of them identified for the first time. Another two candidates turned out to be an emission-line galaxy and an $\mathrm{H}$ in region. All observed PNe represent evolved stages, their angular diameter ranging from $8^{\prime \prime}$ to $90^{\prime \prime}$, and exhibit very low surface brightnesses. The three largest and faintest nebulae each host a central star of magnitude 18 to $20^{\mathrm{m}}$.
\end{abstract}

Key words: planetary nebulae: general

\section{Introduction}

Planetary nebulae (PNe) in late evolutionary stages a long time defied thorough spectroscopic and imaging investigations due to their low or extraordinarily faint surface brightnesses. Correspondingly, there was no traceable continuous emission and, above all, a lack of sufficiently numerous physically usable emission lines or morphological details. However, with the advent of highly sensitive instrumentation these evolved, sometimes even "senile" nebulae proved to be a valuable source of an unexpectedly rich variety in physical conditions: it was, for example, found that the intrinsically largest ones, preferentially those in the galactic plane, are expanding slower than less evolved ones (Hippelein \& Weinberger 1990); further, that there is a pronounced interaction of many of them with the interstellar medium (ISM), opening up, i.e., new possibilities to study the ISM on a small-scale basis (Tweedy \& Kwitter 1994), and that the identification of new senile PNe and the investigations of their central stars considerably helps to understand details of the evolution processes prevailing in the interesting transition zone between PNe nuclei and white dwarfs (Napiwotzki 1995).

In fact, attempts to detect new evolved PNe around hot white dwarfs have, within the last few years, developed some indications of a discovery contest, fought out by means of large-field CCDs and narrow-band filters. The efforts pay off, as was, e.g., very recently demonstrated by Jacoby \& van de Steene (1995), who were able to identify a large nebula around a PG 1159 star and gave an

Send offprint requests to: F. Kerber, florian.kerber@uibk.ac.at overview for some of the primary motivations to try to locate low-density PNe.

Members of our institute have, for a long time, been successfully engaged in the detection (more than $10 \%$ of the registered galactic $\mathrm{PNe}$ ) and investigation of extended PNe (e.g. Weinberger et al. 1983; Hartl \& Tritton 1985; Ishida \& Weinberger 1987; Melmer \& Weinberger 1990; Tamura \& Weinberger 1995). Some of our objects proved to be among the largest planetary nebulae known to date (e.g. Liebert et al. 1994) or turned out to be intriguing in various other respects like harbouring one of the most evolved stars ever identified within a PN (Bruhweiler \& Feibelman 1993).

The PNe discussed in our paper represent chiefly newly discovered objects not mentioned before except in two meetings (Kerber et al. 1994; Kerber \& Weinberger 1995). There however, with the exception of one object where a discussion of its morphology was provided, almost nothing else was given but a list with coordinates and diameters. With the spectra and images presented in this article we are able to discuss these nebulae on a more solid basis.

\section{Observations}

The observations were made during four runs between February and September 1995 at the observatory of the University of Padova located in Asiago. On all occasions we observed with the $1.82 \mathrm{~m}$ Copernico telescope and a B\&C spectrograph. Gratings of 150 and 300 lines $/ \mathrm{mm}$ (both blazed at $5000 \AA$ ) were used, yielding dispersions of $339 \AA / \mathrm{mm}$ and $169 \AA / \mathrm{mm}$ corresponding to ca. 8 and $4 \AA /$ pixel respectively. The usable spectral range is 4000 
to $7400 \AA$ and 4500 to $6700 \AA$. A TH 7882 CCD camera (580 by 388 pixels, UV-coated) with a pixel size of $23 \mu \mathrm{m}$ was used as detector. The slit width was $250 \mu \mathrm{m}$. The seeing varied between $1 . \prime 8$ and 3".5. Exposure times ranged from 900 to 2700 seconds. The narrow band images have been obtained using a 512 by 512 TK $512 \mathrm{M}$ chip with $27 \mu \mathrm{m}$ pixels. The filters have bandwidths of $45 \AA$ for $\mathrm{H}_{\alpha}$ and $73 \AA$ for [O III] .

The objects observed have all been discovered on POSS I paper copies by visual inspection. They have been found both during dedicated searches for PNe as well as a by-product of a search for galaxies near the galactic plane, the so-called Zone of avoidance $(\mathrm{ZoA})$ (see eg. Lercher et al. 1995). It may seem odd that new objects can still be found on this survey after so many years of intense searches but the discovery of new PNe on the POSS has been continuing at a more or less constant rate for many years now. There are two main reasons for this. First, many objects discovered in recent years do not look like a "textbook" PN but come in a variety of shapes and second, most are nondescript objects of low surface brightness. Therefore these objects can easily be overlooked or be mistaken for plate defects (e.g. Weinberger et al. 1994).

It is particularily noteworthy that this kind of $\mathrm{PNe}$ will likely elude most automatic search algorithms on digitized versions of the POSS, since their surface brightness is too close to the background for being successfully found and classified. That's why visual inspection of POSS or $\mathrm{ESO} / \mathrm{SERC}$ plates will remain a valid approach to finding new objects in years to come.

Despite the very limited spectral information provided by its two colors the POSS can also be used for classification of new objects with a very acceptable rate of success. This is illustrated by the fact that 9 out of 11 candidates spectroscopically investigated turned out to be PNe with the remaining two being an $\mathrm{HII}$ region and an emission-line galaxy.

Table 1 summarizes some basic data of the PNe. In the first column we give the common name, in the second the designation following the recommendations outlined in the Strasbourg-ESO Catalogue of Planetary Nebulae (Acker et al. 1992). Columns 3 and 4 give equatorial coordinates, measured from the POSS prints given in Col. 6, accurate to about 6 arcsec. The coordinates have been measured using a high resolution digitizer and software developed at the institute. In Col. 5 a diameter is given as measured on the POSS-E print. Columns 7 and 8 give $x$ and $y$ positions on the POSS prints measured from the lower left corner in $\mathrm{mm}$.

\section{Results and discussion}

In this section we present the observed spectra with the flux given in units of $10^{-14} \mathrm{erg} \mathrm{cm}^{-2} \mathrm{~s}^{-1} \AA^{-1}$. The standard stars used for calibration were GD140 and BD $26^{\circ} 2606$ taken from Massey et al. (1988) and Oke
\& Gunn (1983). The lines of $\mathrm{H}_{\alpha} 6563 \AA$ and [N II] 6584, $6548 \AA$ cannot be separated with the 150 lines $/ \mathrm{mm}$ grating. We have attempted to deconvolve this blend from its profile. Tests show that fluxes for $\mathrm{H}_{\alpha}$ and [N II] deduced in this manner are accurate to better than $25 \%$. The images are reproductions from POSS I paper copies or POSS II films when available. For five objects narrow band images in $\mathrm{H}_{\alpha}$ and [O III] have been obtained. In all images north is up and east to the left.

The spectra of KLW 1 to 3 (Figs. 1 to 3 ) show a very similar characteristic, strong emission in $\mathrm{H}_{\alpha}$ but very faint lines in $[\mathrm{OIII}]$, in fact there are no strong lines in the blue at all. In particular no $\mathrm{H}_{\beta}$ lines could be identified. Given the fact that these three objects are located within a few degrees of one another and within two degrees of the galactic plane, we conclude that these three objects suffer from heavy extinction. Data from Neckel \& Klare (1980) show a linear increase in the reddening in this area to a value of $A_{v} \geq 2.5$ at $1 \mathrm{kpc}$, markedly higher than in the surrounding areas. Their appearance on the POSS is also quite similar to one another; all are small roundish objects of low surface brightness. KLW 2 is an object that warrants further investigation: as can easily be seen the blend of $\mathrm{H}_{\alpha}$ and $[\mathrm{NII}]$ is asymmetric, our analysis suggests a ratio $\mathrm{H}_{\alpha} /[\mathrm{N}$ II] of $\approx 0.5$. KLW 3 shows a pronounced bipolarity on the POSS II B plate. Considering the large extinction it is not surprising that no central stars are apparent.

The continuum of KLW 4 (Fig. 4) stems from a star probably not the central star - that is superimposed on the PN.

KLW 5 (Fig. 5) has a remarkable spectrum. Despite a good signal to noise ratio only very few lines can be seen. It is also apparent that no [N II] lines can be found down to at least the $5 \%$ level of $\mathrm{H}_{\alpha}$. Further investigation is underway to clarify the situation.

KLW 6 (Fig. 6) is a fine example of a spherical shell on the POSS. A preliminary value of 0.7 has been derived for the extinction coefficent. From the ratio $(I(4959)+I(5007)) / 10 I\left(\mathrm{H}_{\beta}\right)$ we conclude that the nebula is of medium excitation (class 4 to 5), as defined by Aller (1956).

For KLW 7 (Fig. 7) we derive $c \approx 1.7$ after deblending $\mathrm{H}_{\alpha}$ and $[\mathrm{NII}]$ as described above. The ratio $(I(4959)+I(5007)) / 10 I\left(\mathrm{H}_{\beta}\right)$ yields an excitation class of 5 . The $\mathrm{H}_{\alpha}$ image shows a slightly elongated disk of homogeneous brightness with only a small enhancement on one side. In [O III] the object looks similar but the disk has a mottled appearance.

The POSS image of KLW 8 (Fig. 8) reveals two distinct lobes superimposed on a homogeneous disk.

KLW 9 (Fig. 9) has an extended brightness enhancement in the northern part of the nebula. We tentatively identify KLW 9 with IRAS $00592+6530$ on grounds of positional agreement $\left(\approx 3^{\prime \prime}\right)$. The flux ratio $F_{\nu}(25) / F_{\nu}(60)$ as measured by IRAS has a value of 0.11 . For $\mathrm{PNe}$ 
Table 1. Basic data for the PNe investigated

\begin{tabular}{|c|c|c|c|c|c|c|c|c|}
\hline Name & Designation & RA (2000.0) & DEC (2000.0) & $\varnothing\left[^{\prime \prime}\right]$ & POSS & $x[\mathrm{~mm}]$ & $y[\mathrm{~mm}]$ & Remarks \\
\hline KLW 1 & PN G051.5+00.2 & $19^{\mathrm{h}} 25^{\mathrm{m}} 37.0^{\mathrm{s}}$ & $+16^{\circ} 36^{\prime} 18^{\prime \prime}$ & 12 & 793 & 81 & 84 & \\
\hline KLW 2 & PN G053.0+01.7 & $19^{\mathrm{h}} 22^{\mathrm{m}} 54.9^{\mathrm{s}}$ & $+18^{\circ} 42^{\prime} 13^{\prime \prime}$ & 20 & 793 & 118 & 199 & \\
\hline KLW 3 & PN G055.0+01.8 & $19^{\mathrm{h}} 26^{\mathrm{m}} 39.9^{\mathrm{s}}$ & $+20^{\circ} 29^{\prime} 21^{\prime \prime}$ & 15 & 793 & 71 & 295 & \\
\hline KLW 4 & PN G066.1+02.0 & $19^{\mathrm{h}} 49^{\mathrm{m}} 57.6^{\mathrm{s}}$ & $+30^{\circ} 16^{\prime} 14^{\prime \prime}$ & 20 & 275 & 7 & 170 & \\
\hline KLW 5 & PN G069.2+01.2 & $20^{\mathrm{h}} 00^{\mathrm{m}} 39.1^{\mathrm{s}}$ & $+32^{\circ} 28^{\prime} 39^{\prime \prime}$ & 12 & 771 & 185 & 285 & \\
\hline KLW 6 & PN G070.9+02.4 & $19^{\mathrm{h}} 59^{\mathrm{m}} 55.5^{\mathrm{s}}$ & $+34^{\circ} 36^{\prime} 04^{\prime \prime}$ & 20 & 200 & 275 & 70 & excitation class $4-5$ \\
\hline KLW 7 & PN G083.7+05.7 & $20^{\mathrm{h}} 22^{\mathrm{m}} 15.9^{\mathrm{s}}$ & $+47^{\circ} 03^{\prime} 53^{\prime \prime}$ & 15 & 1099 & 230 & 94 & excitation class 5 \\
\hline KLW 8 & PN G104.1-01.4 & $22^{\mathrm{h}} 30^{\mathrm{m}} 10.4^{\mathrm{s}}$ & $+56^{\circ} 11^{\prime} 43^{\prime \prime}$ & 15 & 588 & 6 & 25 & \\
\hline KLW 9 & PN G124.0+02.9 & $01^{\mathrm{h}} 02^{\mathrm{m}} 24.7^{\mathrm{s}}$ & $+65^{\circ} 46^{\prime} 36^{\prime \prime}$ & 15 & 1234 & 167 & 121 & IRAS $00592+6530 ?$ \\
\hline KLW 10 & PN G129.6-05.6 & $01^{\mathrm{h}} 40^{\mathrm{m}} 05.7^{\mathrm{s}}$ & $+56^{\circ} 34^{\prime} 44^{\prime \prime}$ & 90 & 945 & 63 & 273 & $\mathrm{CS} m_{\mathrm{b}}=18.5$ \\
\hline KLW 11 & PN G193.0-04.5 & $05^{\mathrm{h}} 57^{\mathrm{m}} 09.0^{\mathrm{s}}$ & $+15^{\circ} 25^{\prime} 29^{\prime \prime}$ & 60 & 1494 & 320 & 38 & $\mathrm{CS} m_{\mathrm{b}} \approx 20$ \\
\hline KLW 12 & PN G197.0+05.8 & $06^{\mathrm{h}} 43^{\mathrm{m}} 25.6^{\mathrm{s}}$ & $+16^{\circ} 48^{\prime} 42^{\prime \prime}$ & 35 & 1470 & 32 & 116 & $\mathrm{CS} m_{\mathrm{b}}=19$ \\
\hline TaWe 2 & PN G065.4+03.1 & $19^{\mathrm{h}} 43^{\mathrm{m}} 47.9^{\mathrm{s}}$ & $+30^{\circ} 14^{\prime} 08^{\prime \prime}$ & 15 & 275 & 79 & 169 & \\
\hline TaWe 4 & PN G070.8+03.7 & $19^{\mathrm{h}} 54^{\mathrm{m}} 17.3^{\mathrm{s}}$ & $+35^{\circ} 07^{\prime} 08^{\prime \prime}$ & 8 & 200 & 336 & 106 & \\
\hline \multirow[t]{3}{*}{ WSLS 1} & PN G160.7-02.9 & $04^{\mathrm{h}} 37^{\mathrm{m}} 20.3^{\mathrm{s}}$ & $+42^{\circ} 47^{\prime} 15^{\prime \prime}$ & 25 & 644 & 198 & 199 & excitation class $4-5$ \\
\hline & G083.0-08.3 & $21^{\mathrm{h}} 18^{\mathrm{m}} 40.7^{\mathrm{s}}$ & $+37^{\circ} 34^{\prime} 49^{\prime \prime}$ & 13 & 269 & 328 & 228 & emission-line galaxy \\
\hline & $\mathrm{G} 104.9+11.2$ & $21^{\mathrm{h}} 30^{\mathrm{m}} 55.5^{\mathrm{s}}$ & $+66^{\circ} 49^{\prime} 06^{\prime \prime}$ & 100 & 1165 & 237 & 184 & $\mathrm{H}_{\text {II }}$ region \\
\hline
\end{tabular}
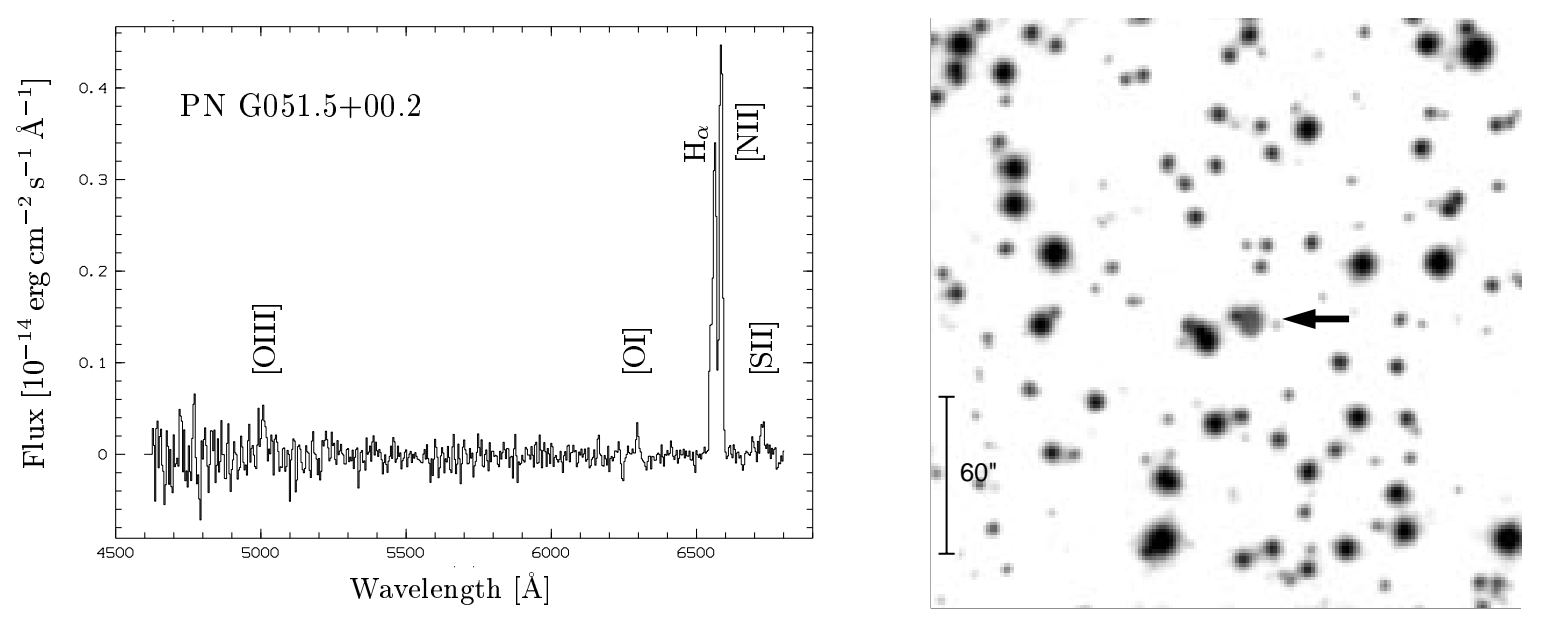

Fig. 1. Spectrum and POSS-image for KLW 1, PN G051.5+00.2
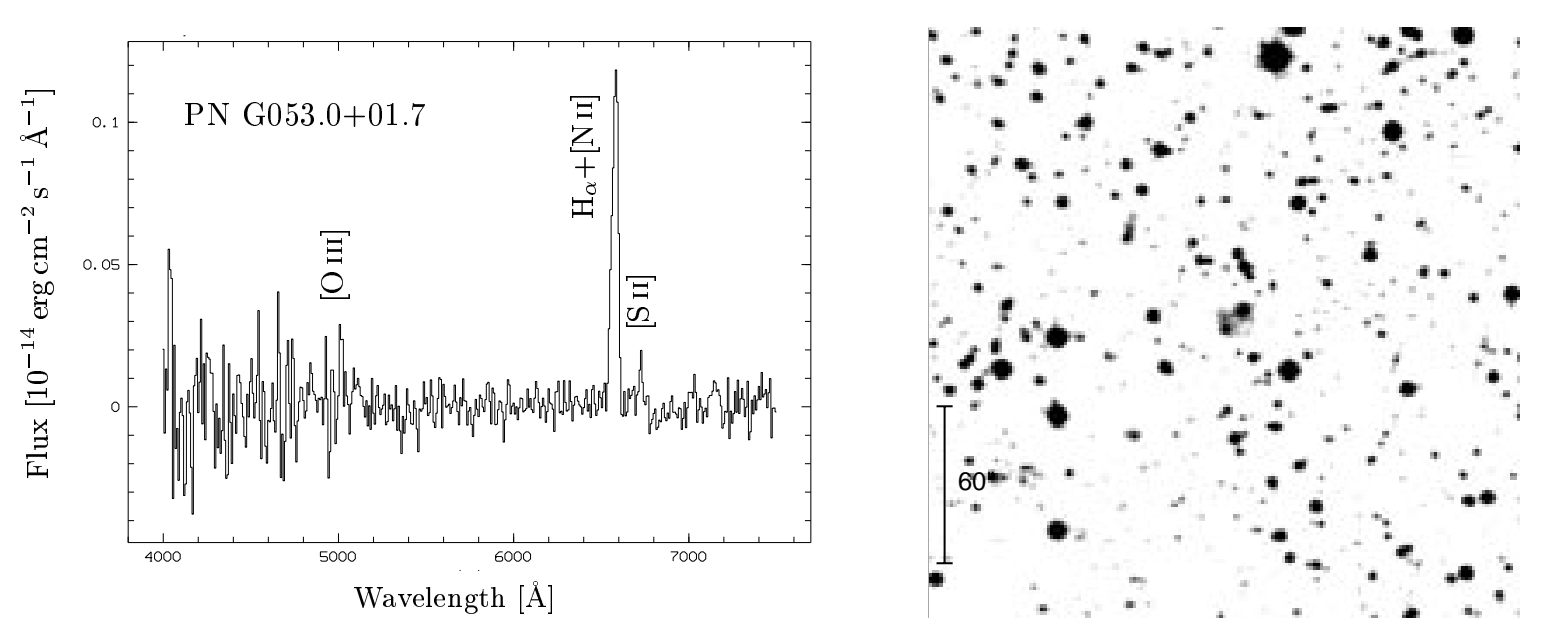

Fig. 2. Spectrum and POSS-image for KLW 2, PN G053.0+01.7 

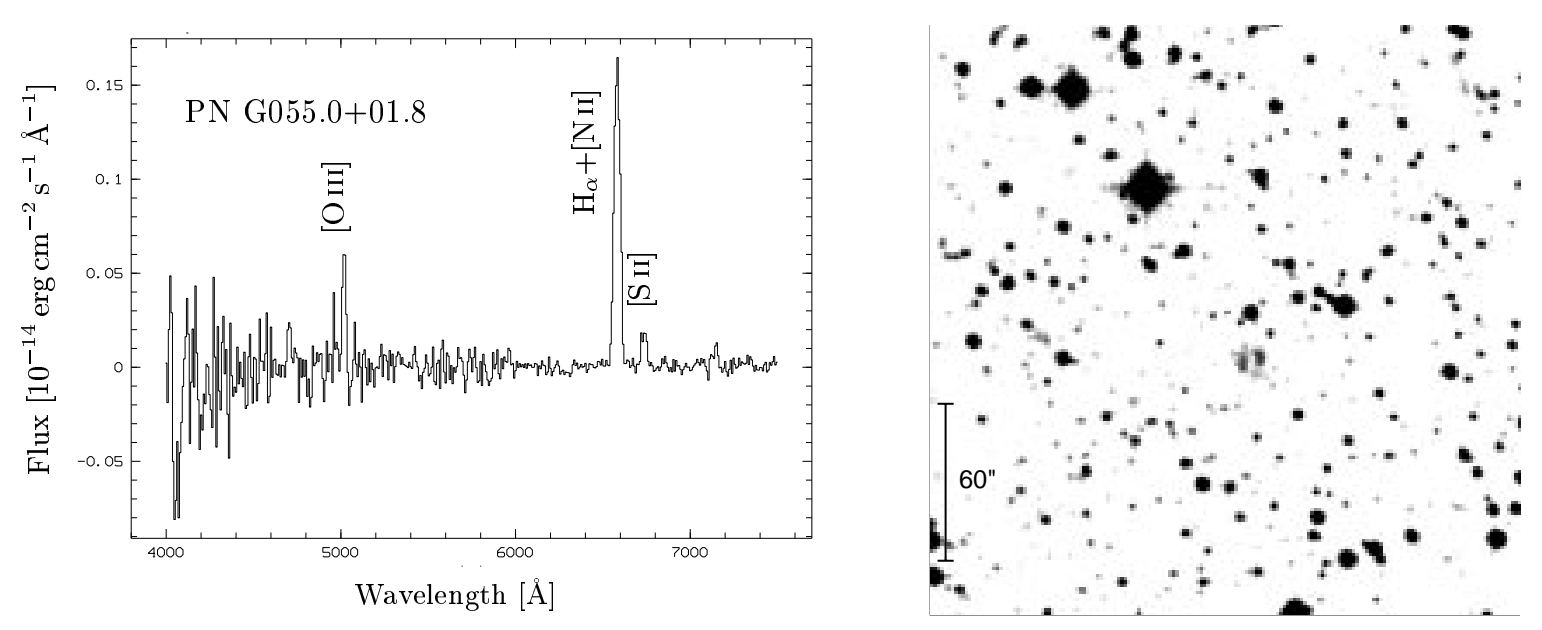

Fig. 3. Spectrum and POSS-image for KLW 3, PN G055.0+01.8
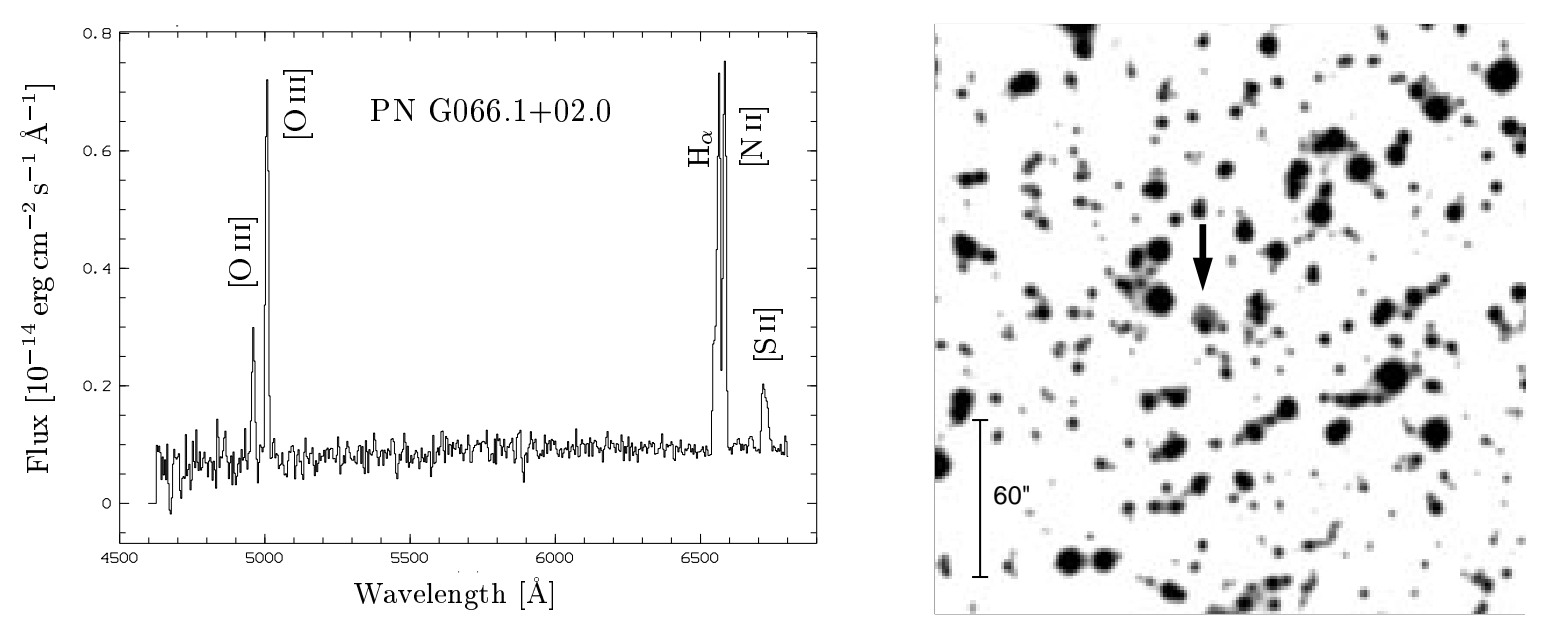

Fig. 4. Spectrum and POSS-image for KLW 4, PN G066.1+02.0
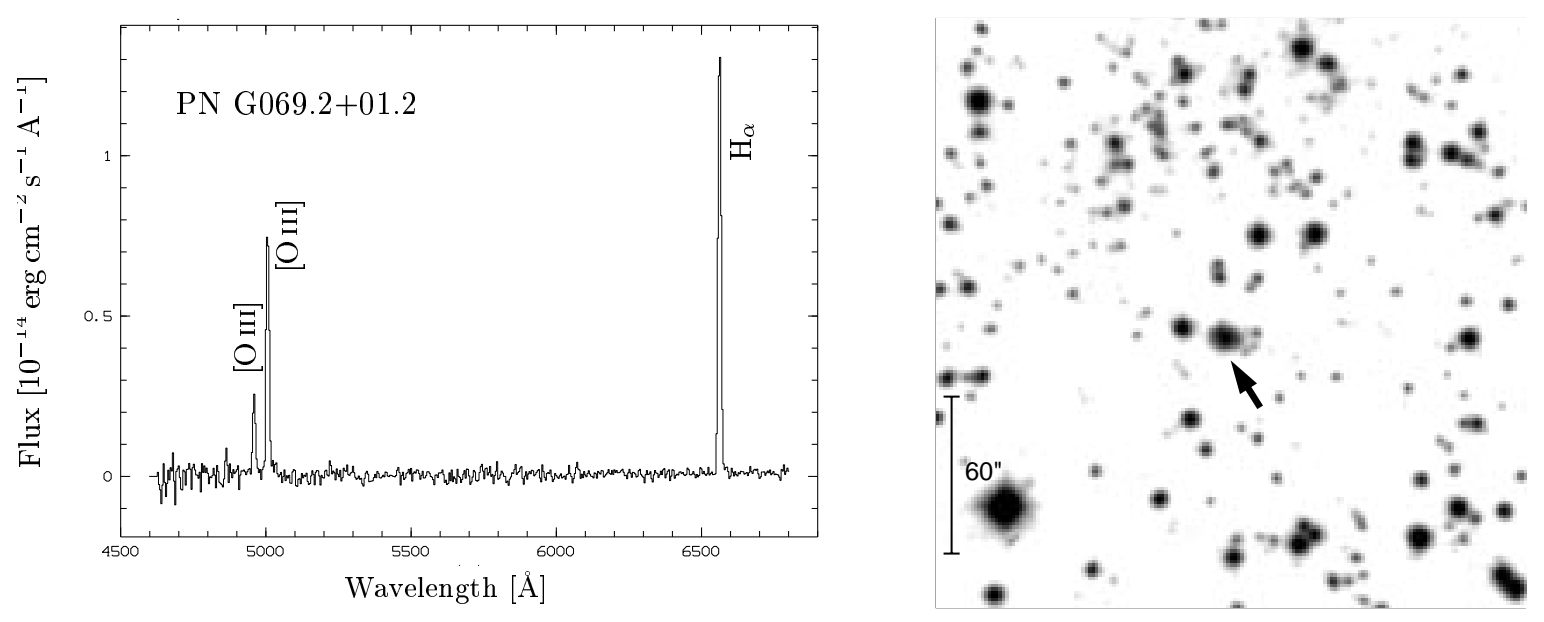

Fig. 5. Spectrum and POSS-image for KLW 5, PN G069.2+01.2 

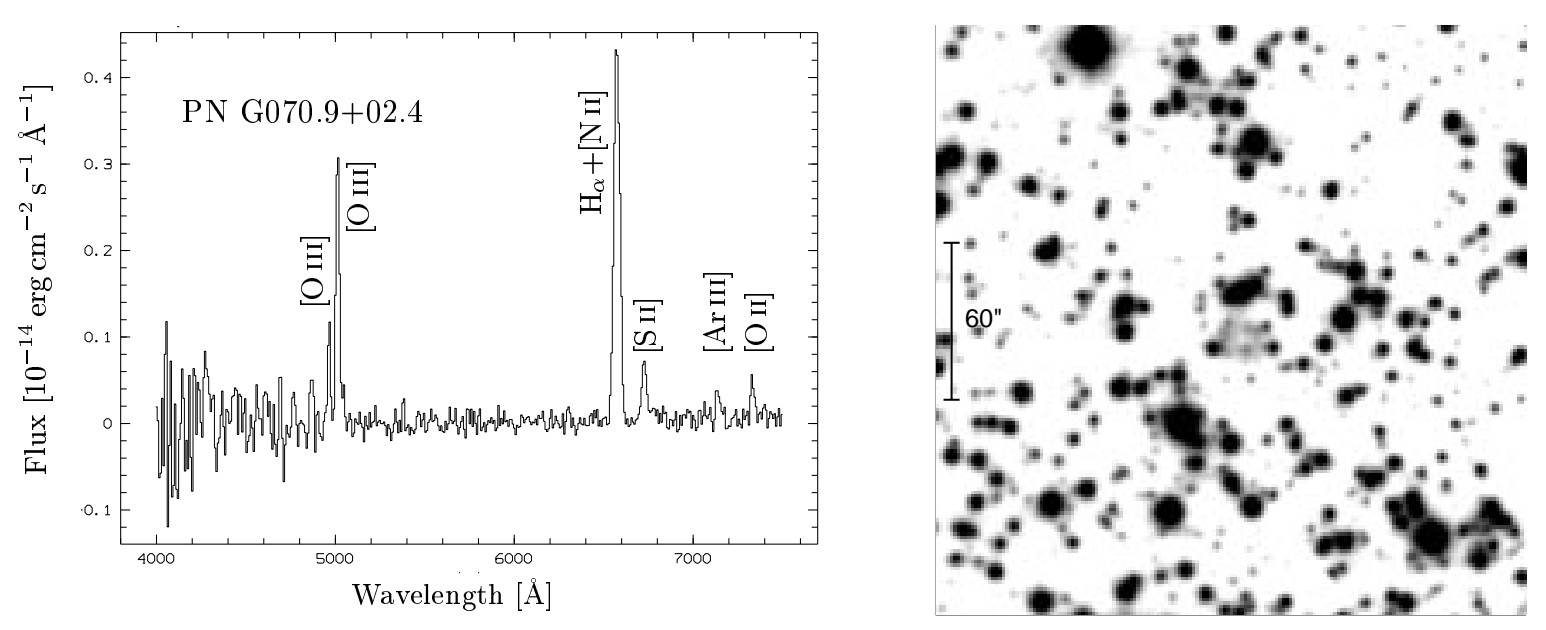

Fig. 6. Spectrum and POSS-image for KLW 6, PN G070.9+02.4
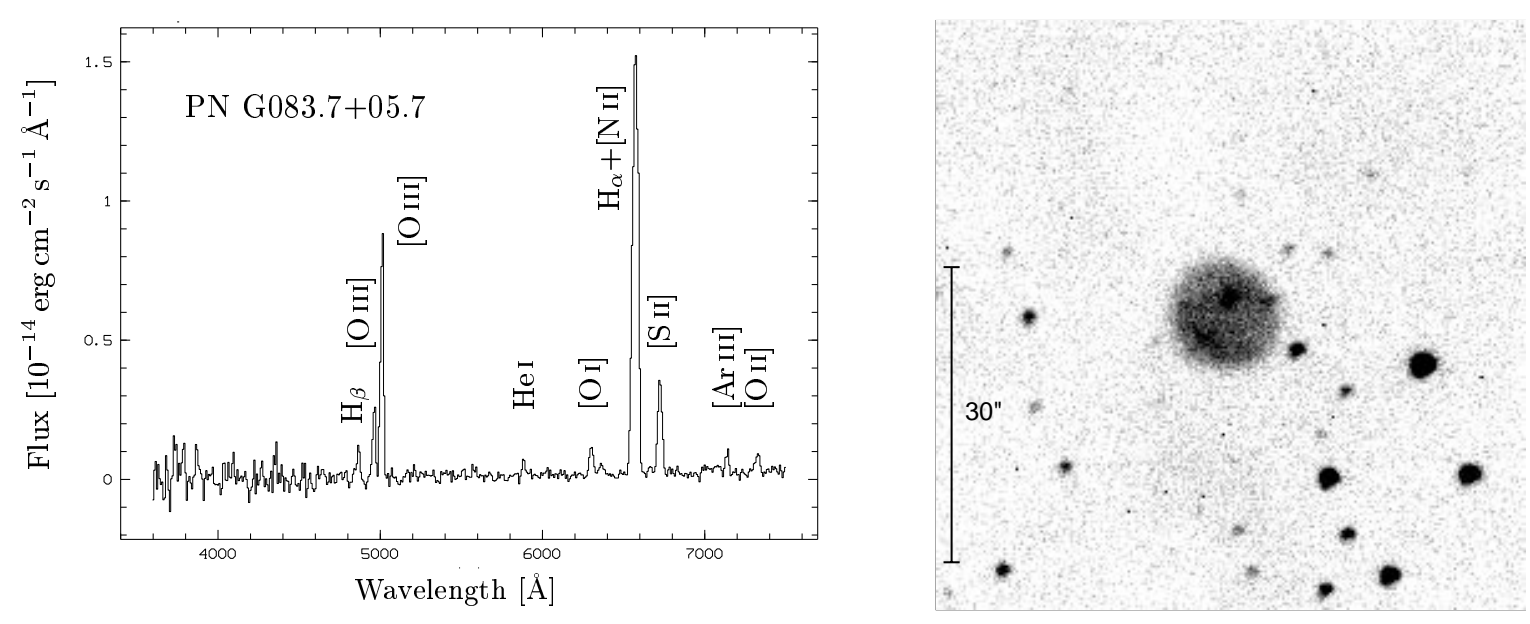

Fig. 7. Spectrum and $\mathrm{H}_{\alpha}$-image for KLW 7, PN G083.7+05.7
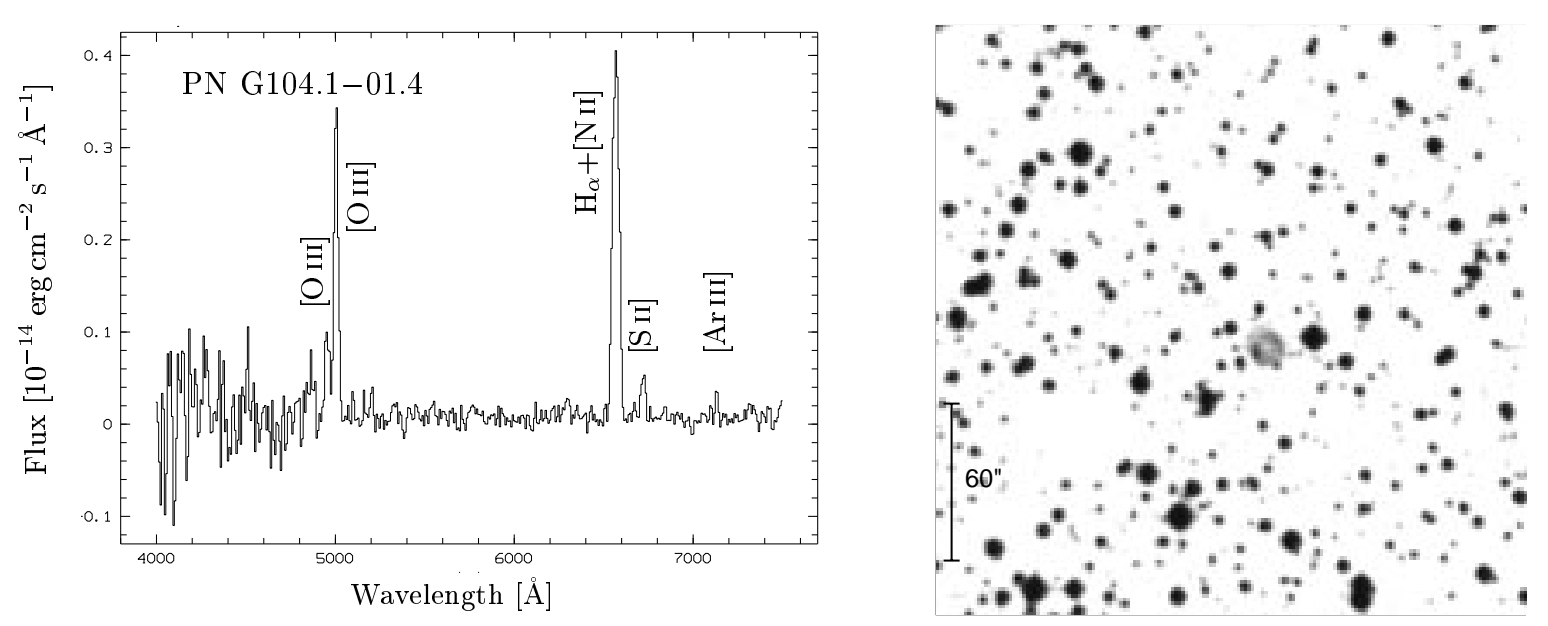

Fig. 8. Spectrum and POSS-image for KLW 8, PN G104.1-01.4 

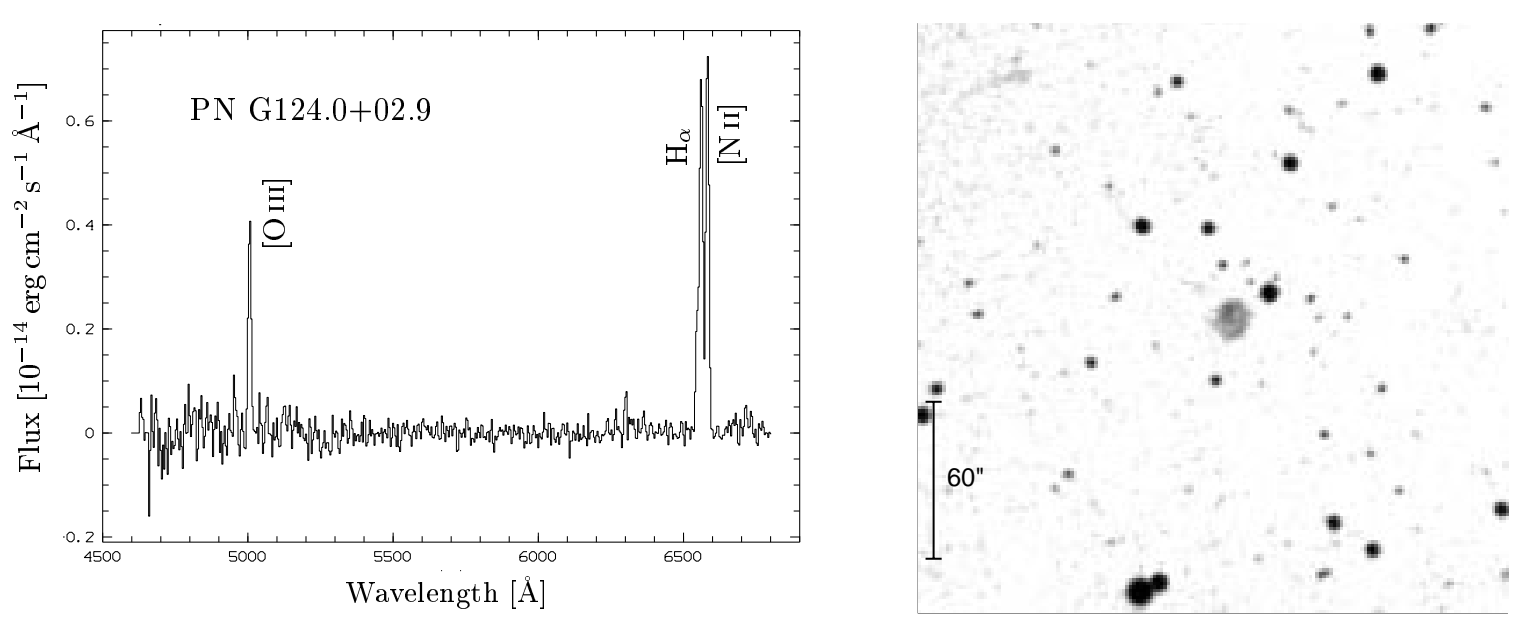

Fig. 9. Spectrum and POSS-image for KLW 9, PN G124.0+02.9
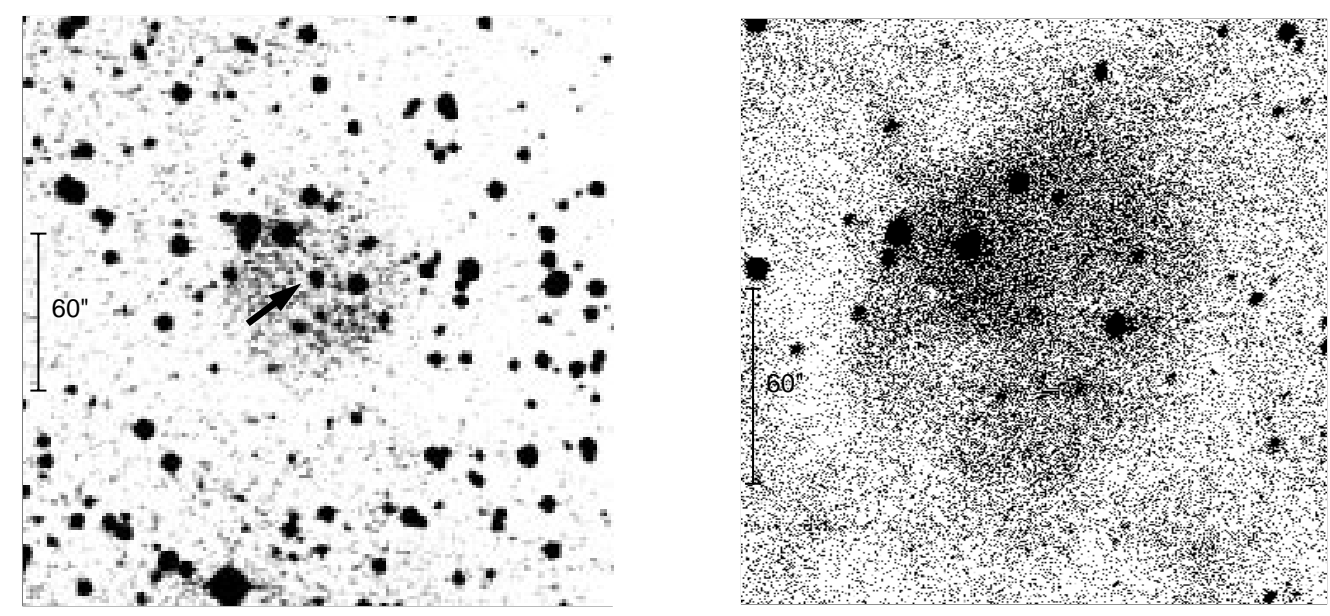

Fig. 10. POSS-B and $\mathrm{H}_{\alpha}$ images for KLW 10, PN G129.6-05.6. The central star is marked
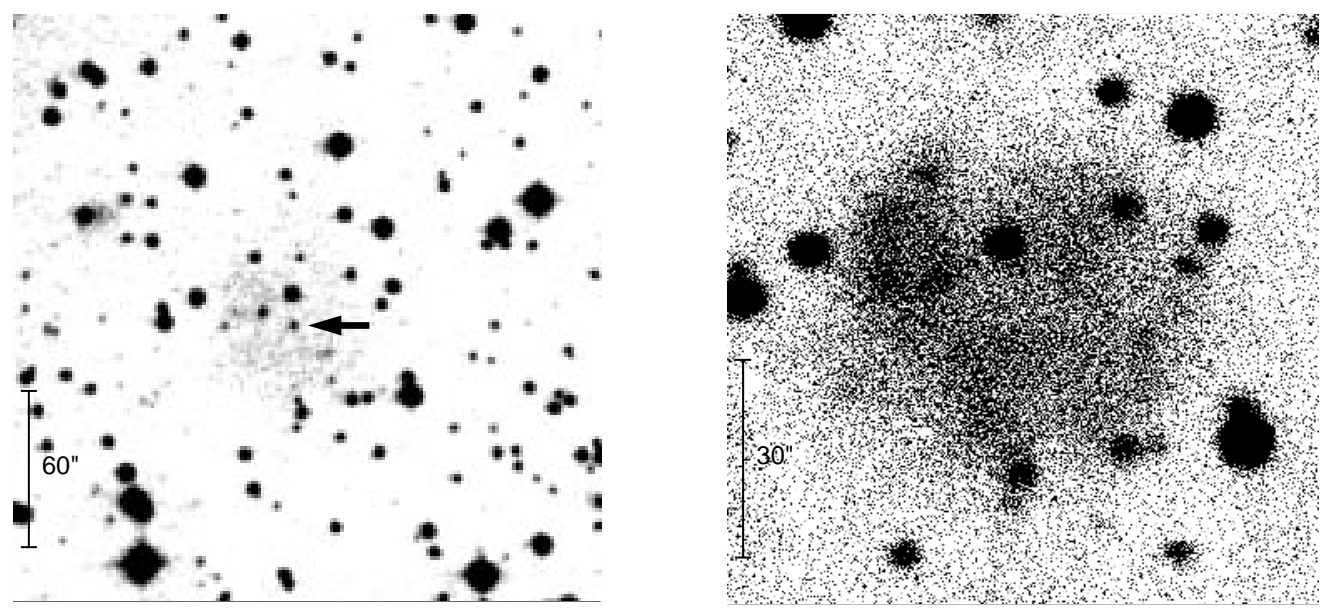

Fig. 11. POSS-B and $H_{\alpha}$ images for KLW 11, PN G193.0-04.5. The central star is marked 

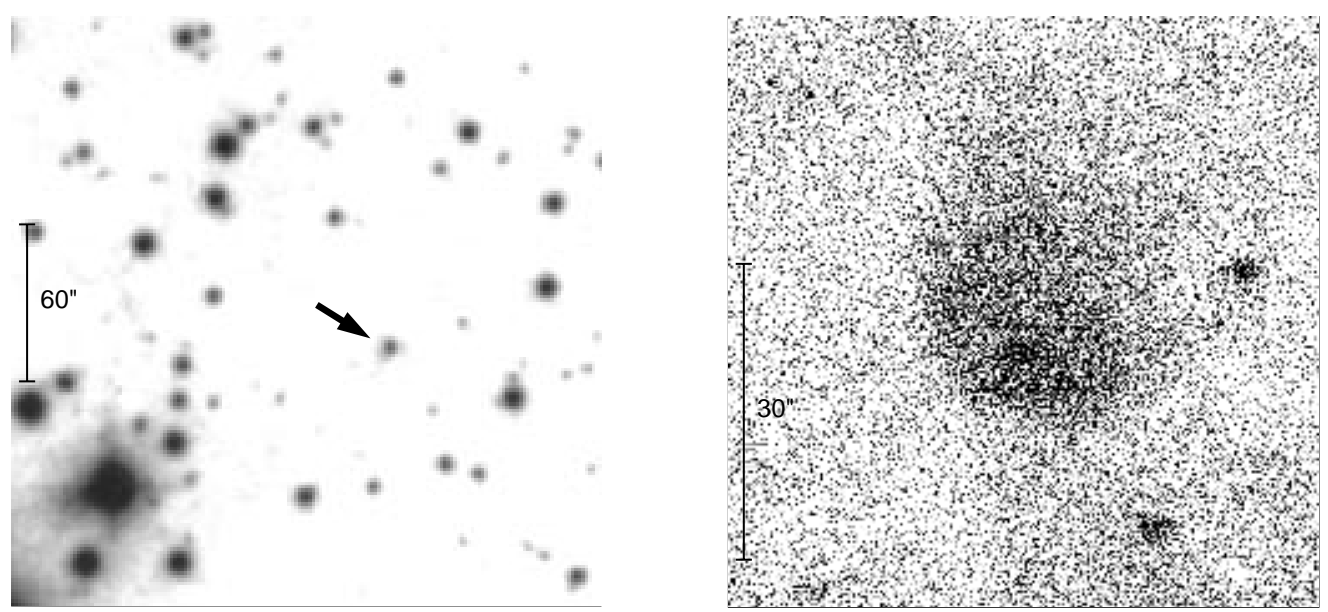

Fig. 12. POSS-O and $\mathrm{H}_{\alpha}$ images for KLW 12, PN G197.0+05.8. The central star is marked
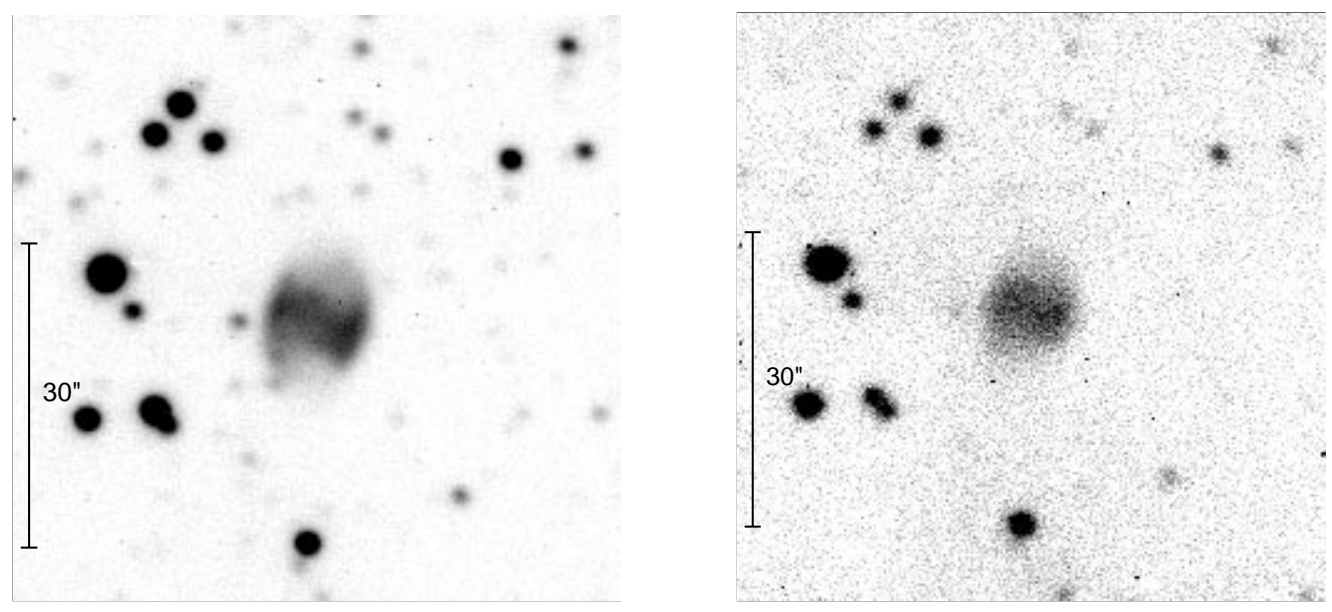

Fig. 13. $\mathrm{H}_{\alpha}$ and $\left[\mathrm{O}_{\text {III }}\right]$ images for TaWe 2, PN G065.4+03.1
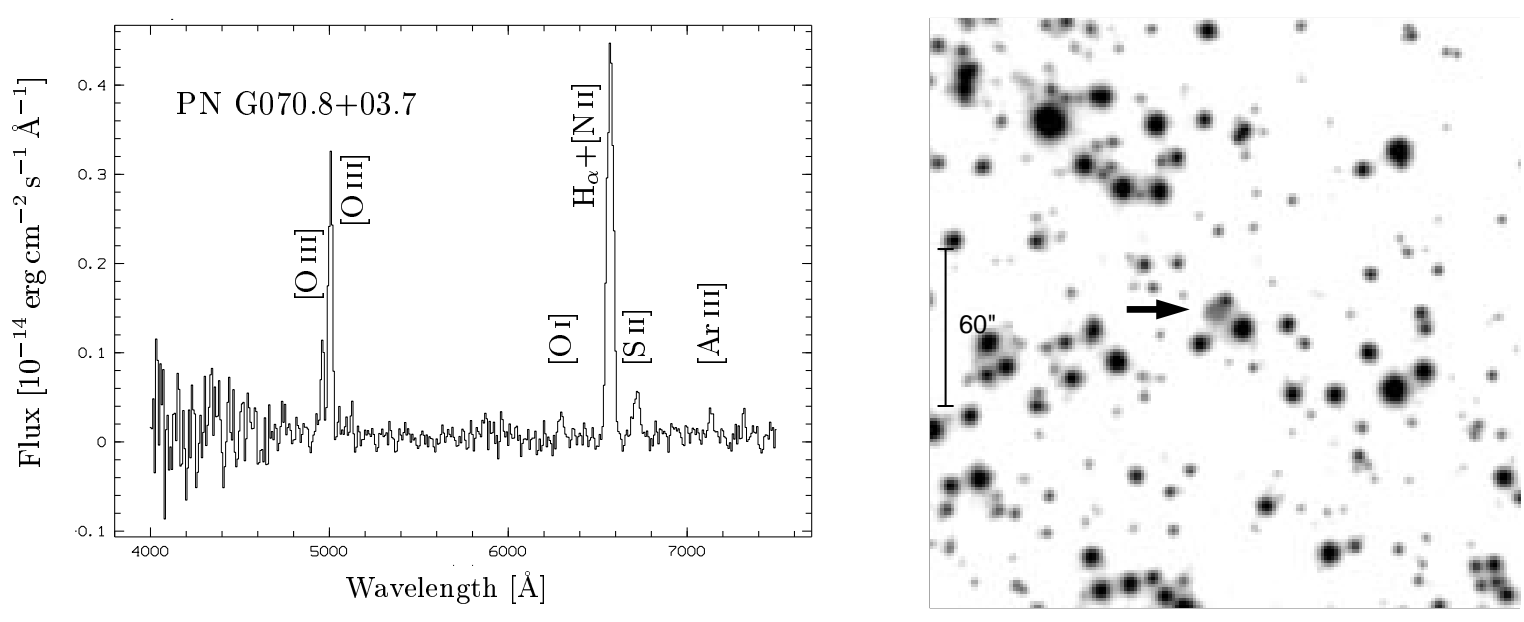

Fig. 14. Spectrum and POSS-image for TaWe4, PN G070.8+03.7 
values larger than 0.3 are expected, so the physical identity remains uncertain.

For KLW 10 to 12 (Figs. 10 to 12) we could identify a central star. For KLW 10 and KLW 11 only a mere hint of a nebula can be seen on the POSS I but a faint distinctly blue star was found. The POSS II revealed a roundish nebula of extremely low surface brightness surrounding the star. The $\mathrm{H}_{\alpha}$ images confirm that both are emission line objects. For KLW 12 no POSS II films were available but a faint nebula showed up in $\mathrm{H}_{\alpha}$. All three objects have an apparently very faint distinctly blue star. Their colour means that reddening and therefore extinction can only be small towards these objects. This again makes the stars intrinsically blue and intrinsically faint. The nebulae cannot be $\mathrm{H}$ II regions since the exciting stars of the latter would be more luminous. We conclude that objects KLW 10, 11 and 12 are most likely PNe despite the fact that no spectrum has been obtained, yet.

The $\mathrm{H}_{\alpha}$ and [OIII] (Fig. 13) images for TaWe,2 show an elliptical nebula and a central bar with brightness enhancements at its ends.

For WSLS 1 a spectrum has been published already, but no flux calibration was possible at that time, see Weinberger et al. (1994). This has been accomplished here and a value of $\approx 0,2$ for $c$ has been deduced from the Balmer decrement. The nebula is of medium excitation (class 4 - 5).

For TaWe 4 (Fig. 14) our spectrum confirms the conjecture by Tamura \& Weinberger (1995) that this object is markedly reddened; no $\mathrm{H}_{\beta}$ was detected.

The new objects presented here, nicely demonstrate that many more relatively large and therefore close $\mathrm{PNe}$ remain to be found, before a reliable number can be derived for the local PN sample and therefore for the galactic PNe population as a whole. Interestingly a possible IRAS counterpart has been found for only one out of twelve candidates, supporting the notion that these objects are in a highly evolved state. It may also be noted that new detections of (extended) PNe are not only interesting from a statistical point of view, but are also of considerable significance due to the extreme heterogeneity of the class: each $\mathrm{PN}$ is, to a certain extent, unique. Photographic surveys like the (old) POSS I or the ESO/SERC atlas were by far the most fruitful material for the search for evolved $\mathrm{PNe}$ and hundreds of new planetaries were discovered by scouring them for new candidates. Curiously, although examined and reexamined by several researchers, these surveys are obviously still not exhausted of their riches: this very article is a compelling evidence for such a statement.

Acknowledgements. Special thanks are due to the University of Padova for generously granting observing time and to the members of the staff at Cima Ekar for their assistance. We greatfully acknowledge the financial support by the "Fonds zur Förderung der wissenschaftlichen Forschung"; project P10279AST. This research has made use of the Simbad database, operated at CDS, Strasbourg, France.

\section{References}

Acker A., Ochsenbein F., Stenholm B., Tylenda R., Marcout J., Schon C., 1992, Strasbourg-ESO Catalogue of Galactic Planetary Nebulae

Aller L.H., 1956, Gaseous Nebulae, p. 66

Bruhweiler F.C., Feibelman W.A., 1993, AJ 105, 1477

Hartl H., Tritton S.B., 1985, A\&A 145, 41

Hippelein H., Weinberger R., 1990, A\&A 232, 129

Ishida K., Weinberger R., 1987, A\&A 178, 227

Jacoby G.H., Van de Steene, G., 1995, AJ 110, 1285

Kerber F., Weinberger R., 1995, Ann. Israel Phys. Soc. 11, 193

Kerber F., Lercher G., Saurer W., Seeberger R., Weinberger R., 1994, AG Abst. Ser. 10, 172

Lercher G., Kerber F., Weinberger R., 1996, A\&AS 117, 369

Liebert J., Bergeron P., Tweedy R.W., 1994, ApJ 424, 817

Massey P., Strobel K., Barnes J.V., Anderson E., 1988, ApJ 328,315

Melmer D., Weinberger R., 1990, MNRAS 243, 236

Napiwotzki R., 1995, Proc. on White Dwarfs, Kiel 1994, Lect. Notes Phys., 176

Neckel Th., Klare G., 1980, A\&AS 42, 251

Oke J.B., Gunn J.E., 1983, ApJ 266, 713

Reich W., 1994, A\&A 290, 639

Tamura S., Weinberger R., 1995, A\&A 298, 204

Tweedy R.W., Kwitter K.B., 1994, AJ 108, 188

Weinberger R., Saurer W., Lercher G., Seeberger R., 1994, A\&A 282, 198

Weinberger R., Dengel J., Hartl H., Sabbadin F., 1983, ApJ 265,249 